\title{
The complete chloroplast genome sequence of Korean Neolitsea sericea (Lauraceae)
}

\author{
Yoo-Jung PARK and Kyeong-Sik CHEON*
}

Department of Biological Science, Sangii University, Wonju 26339, Korea

(Received 10 June 2021; Revised 13 July 2021; Accepted 27 August 2021)

\begin{abstract}
The complete chloroplast (cp) genome sequence of Neolitsea sericea was determined by Illumina sequencing. The complete cp genome was $152,446 \mathrm{bp}$ in length, containing a large single-copy region of 93,796 bp and a small single-copy region of $18,506 \mathrm{bp}$, which were separated by a pair of 20,072 bp inverted repeats. A total of 112 unique genes were annotated, including 78 protein-coding genes (PCGs), 30 transfer RNAs, and four ribosomal RNAs. Among the PCGs, 18 genes contained one or two introns. A very low level of sequence variation between two cp genomes of $N$. sericea was found with seven insertions or deletions and only one single nucleotide polymorphism. An analysis using the maximum likelihood method showed that $N$. sericea was closely related to Actinodaphne trichocarpa.
\end{abstract}

Keywords: Lauraceae, Neolitsea sericea, chloroplast genome, intraspecific variation

The genus Neolitsea (Benth. \& Hook.f.) Merr. (Lauraceae) consists of approximately 85 species of dioecious trees or shrubs and is distributed throughout tropical and subtropical Asia and Australia (Li et al., 2007; Qing et al., 2014). Among these species, Neolitsea sericea (Blume) Koidz., discussed in this study, was initially described as Laurus sericea (Blume, 1826) but has since been treated as a species belonging to the genus Neolitsea (Koidzumi, 1926). This species is an evergreen broadleaf tree and dioecious. The leaves are verticillate and elliptical with three pairs of lateral veins, and the flowers are yellowish white forming umbellate clusters without peduncle at the leaf axils. The fruit is a drupe and ripens to red in October of the following year. This species is very similar to $N$. aurata (Hayata) Koidz. and N. lunglingensis $\mathrm{H}$. W. Li, but distinguished from these species in that the leaf blade is shortly acuminate and the fruit is globose (Huang and Werff, 2008; Kim, 2019).

Neolitsea sericea is distributed in China, Korea and Japan and is commonly found in the evergreen forests of Korea and Japan. The Korean Peninsula and the main island of Japan are also recognized as important regions because they represent the northern-most limit of its range (Ohashi et al., 2006; Lee and Choi, 2010; Lee et al., 2013). Due to these distributional characteristics, this species is recognized as a major indicator of climate change on the Korean Peninsula (Yun et al., 2014). In addition, the species is an important plant resource used as ornamental trees, timber, and in the creation of natural dyes and essential oils as treatments for inflammatory diseases (Jo et al., 2007; Yoon et al., 2010).

The first chloroplast (cp) genome sequence of this species was reported by Song et al. (2017) and was isolated in Zhejiang Province in China. In this study, we report the second complete chloroplast genome sequence of $N$. sericea from a sample collected on Jejudo Island in Korea. We also carry out comparative analyses between two plastomes to recognize the infraspecific variation.

\section{Materials and Methods}

\section{Plant material}

The plant materials were sampled from Bonggae-dong $\left(33^{\circ} 25^{\prime} 37.8^{\prime \prime N}, 126^{\circ} 38^{\prime} 39.2^{\prime \prime E}\right)$, Jeju-si, Jeju-do, and a voucher specimen was deposited in the Sangji University Herbarium (SJUH; Kyeong-Sik Cheon, cheonks@sangji.ac.kr), with voucher number SJUH000861.

\footnotetext{
*Author for correspondence: cheonks@sangji.ac.kr
} 


\section{DNA extraction and cp genome determination}

Total DNA was extracted from fresh leaf using a DNeasy Plant Mini Kit (Qiagen Inc., Valencia, CA, USA). The Pairedend library was constructed with a TruSeq DNA Sample Preparation Kit (Illumina Inc., San Diego, CA, USA). Genomic DNA sequencing was performed using the Illumina MiSeq (Illumina Inc.) platform to produce 3,438,412 raw reads with a length of $301 \mathrm{bp}$.

The assembly and annotation of the cp genome were accomplished using the Geneious Prime v2020.1.3 (Biomatters Ltd, Auckland, New Zealand). For correct gene annotation, we also compared each gene to four published complete chloroplast genomes of Lauraceae: Actinodaphne trichocarpa C. K. Allen (GenBank accession no. MF939342), Lindera pulcherrima (Nees) Hook.f. (GenBank accession no. MN453268), L. chunii Merr. (GenBank accession no. NC045254), and L. aggregata
(Sims) Kosterm. (GenBank accession no. NC045252). The tRNAs were confirmed using tRNAscan-SE v2.0 (Chan and Lowe, 2019). A circle cp genome map was drawn using the OGDRAW program (Greiner et al. 2019).

\section{Phylogenetic analysis}

To construct the phylogenetic tree, a total of 74 protein-coding genes (PCGs) of 50 species were aligned using the MAFFT (v7.450) program (Katoh and Standley, 2013). Forty-eight Lauraceae accessions were selected as the ingroups, and two Calycanthaceae species, Chimonanthus nitens Oliv. (MH377057) and C. praecox (L.) Link (MH377058), were chosen as outgroups. We selected the GTR $+\mathrm{I}+\Gamma$ model as the best substitution model according to jModelTest version 2.1.10 (Darriba et al., 2012). Maximum likelihood (ML) analyses were performed using PAUP* 4.0a168 (Swofford, 2002) with 1,000 bootstrap replicates.

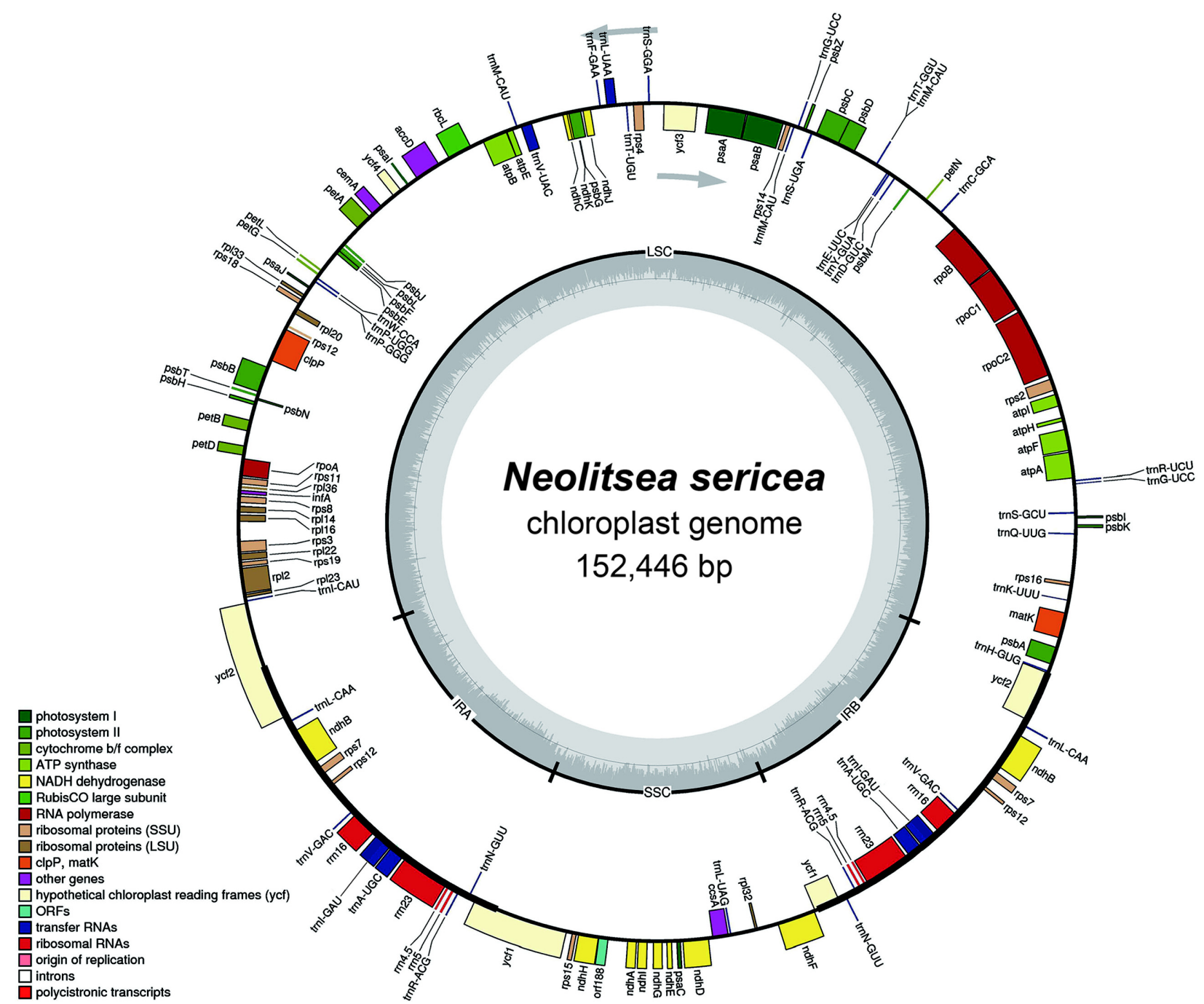

Fig. 1. Structural map of the chloroplast genome in Neolitsea sericea. Genes inside the circle are transcribed clockwise, genes outside are transcribed counter-clockwise. The dark gray inner circle corresponds to the GC content, the light-gray to the AT content. 


\section{Results and Discussion}

A circular form of the complete chloroplast genome of $N$. sericea (MW465540) is $152,446 \mathrm{bp}$ in length with $39.2 \% \mathrm{G}+\mathrm{C}$ content, composed of a large single-copy (LSC) region of 93,796bp, a small single-copy (SSC) region of 18,506bp, and two inverted repeat (IR) regions of 20,072bp (Fig. 1). The whole genome of Korean $N$. sericea was 4 bp longer than that of the first cp genome $(152,442 \mathrm{bp})$; the LSC region was $7 \mathrm{bp}$ shorter, SSC region was 1 bp longer, and the IR regions were

Table 1. Sequence variation between the two chloroplast genomes of Neolitsea sericea.

\begin{tabular}{|c|c|c|c|c|c|}
\hline \multirow[b]{2}{*}{ No. } & \multicolumn{2}{|c|}{ GenBank accession number of chloroplast genomes } & \multirow{2}{*}{$\begin{array}{l}\text { Aligned position } \\
\text { (bp) }\end{array}$} & \multirow[b]{2}{*}{ Gene } & \multirow[b]{2}{*}{ Region } \\
\hline & $\begin{array}{c}\text { MF939341 } \\
\text { (Song et al., 2017) }\end{array}$ & $\begin{array}{l}\text { MW465540 } \\
\text { (this study) }\end{array}$ & & & \\
\hline 1 & $\mathrm{~T}$ & - & 10,578 & $\operatorname{trn} G-U C C$ (intron) & LSC \\
\hline 2 & - & A & 15,160 & atpH-atpI & $\mathrm{LSC}$ \\
\hline 3 & A & - & 38,968 & 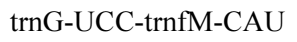 & LSC \\
\hline 4 & A & - & 38,969 & trnG-UCC-trnfM-CAU & LSC \\
\hline 5 & $\mathrm{~A}$ & - & 47,866 & $\operatorname{trnS-GGA-rps4}$ & LSC \\
\hline 6 & - & $\mathrm{T}$ & 66,643 & petA-psbJ & LSC \\
\hline 7 & $\mathrm{~T}$ & $\mathrm{C}$ & 71,234 & psaJ-rpl33 & LSC \\
\hline 8 & - & A & 116,703 & ndhF-rpl32 & SSC \\
\hline
\end{tabular}

LSC, large single-copy; SSC, small single-copy.

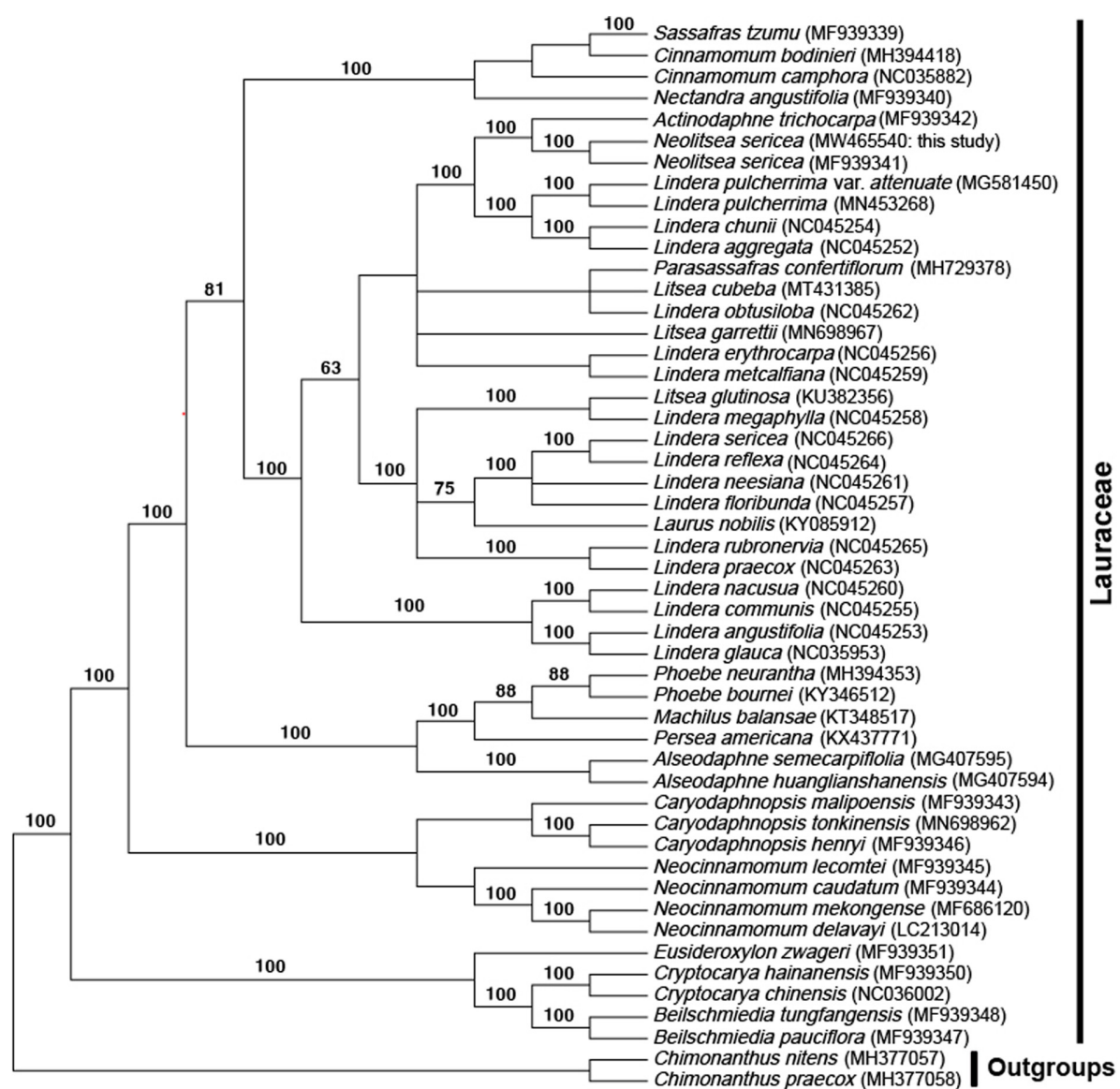

Fig. 2. The maximum likelihood tree based on 74 protein-coding genes from 48 Lauraceae species and two outgroups. Numbers on branches indicate bootstrap value greater than $50 \%$. 
5 bp longer. Also, the LSC region of the second cp genome was $7 \mathrm{bp}$ shorter, and its SSC and IR regions were $1 \mathrm{bp}$ and 5 bp longer than the corresponding regions of the Chinese cp genome (MF939341). The plastid genome contains a total of 112 unique genes consisting of 78 PCGs, 30 transfer RNAs, and four ribosomal RNAs. Additionally, twelve complete genes are duplicated in the IR regions. Among the 112 unique genes, eighteen (atpF, clpP, ndhA, ndhB, petB, petD, rps12, rpl16, rpoC1, rps12, rps16, ycf3, trnA-UGC, trnG-UCC, trnI-GAU, $\operatorname{trnK}-U U U, \operatorname{trnL}-U A A$, and $\operatorname{trn} V-U A C)$ contained one or two introns.

Based on the pairwise alignment of the two cp genomes, seven insertions or deletions (INDELs) and one single nucleotide polymorphism (SNP) were identified (Table 1). Among these, six indels and one SNP were in the LSC, and only one INDEL was correspondingly identified in the SSC. The infraspecific cp genome variation in this species is significantly lower than those in other land plants: 121 SNPs and 781 INDELs in Pyrus ussuriensis Maxim. (Cho et al., 2019), 84 SNPs and 125 INDELs in Pseudostellaria palibiniana Takeda (Kim et al., 2019), 69 SNPs and 660 INDELs in Marchantia polymorpha L. (Kwon et al., 2019), 78 SNPs and 643 INDELs in Camellia japonica L. (Park et al., 2019), 16 SNPs and 49 INDELs in Viburnum erosum Thunb. (Choi et al., 2020), 258 SNPs and 542 INDELs in Agrimonia Pilosa Ledeb. (Heo et al., 2020), 7 SNPs and 4 INDELs in Veronica nakaiana Ohwi (Lee et al., 2021), 33 SNPs and 662 INDELs in Campanula takesimana Nakai (Park et al., 2021), and 69 SNPs and 650 INDELs in Daphne genkwa Siebold \& Zucc. (Yoo et al., 2021).

The ML tree (Fig. 2) formed five clades. The first clade consisted of the genera Beilschmiedia Nees, Cryptocarya R. Br. and Eusideroxylon Teijsm. \& Binn. and formed the most basal part. The second clade is made up of Neocinnamomum H. Liu and Caryodaphnopsis Airy-Shaw. The third clade and fourth clade consist of four genera (Alseodaphne Nees, Persea Mill., Machilus Nees, and Phoebe Nees) and three genera (Nectandra Rol. Ex Rottb., Cinnamomum Schaeff., and Sassafras L. ex Nees), respectively. The last clade consists of the remaining genera excluding those mentioned above with Lindera (Adans.) Thunb. being paraphyletic. Also, N. sericea is closely related to $A$. trichocarpa with a high support value (bootstrap value $=100$ ).

A comparative analysis of the cp genome sequences between the Korean and Chinese $N$. sericea in this study provides useful information to understanding of the evolution and diversification of this species. This study will provide important information for further studies of the ecology and genetics/genomics of this species.
ORCID: Yoo-Jung Park https://orcid.org/0000-0002-3122-3173; Kyeong-Sik Cheon https://orcid.org/0000-0002-2182-7379

\section{Acknowledgments}

This study was performed with the support of the Sangji University and Graduate School of Sangji University.

\section{Conflict of Interest}

The authors declare that there are no conflicts of interest.

\section{Literature Cited}

Blume, C. L. 1826. Bijdragen tot de flora von Nederlandsch Indië. Ter Lands Drukkerij, Batavia, 554 pp.

Chan, P. P. and T. M. Lowe. 2019. tRNAscan-SE: Searching for tRNA genes in genomic sequences. Methods in Molecular Biology 1962: 1-14.

Cho, M.-S., Y. Kim, S.-C. Kim and J. Park. 2019. The complete chloroplast genome of Korean Pyrus ussuriensis Maxim. (Rosaceae): Providing genetic background of two types of $P$. ussuriensis. Mitochondrial DNA Part B 4: 2424-2425.

Choi, Y. G., N. Yun, J. Park, H. Xi, J. Min, Y. Kim and S.-H. Oh. 2020. The second complete chloroplast genome sequence of the Viburnum erosum (Adoxaceae) showed a low level of intra-species variations. Mitochondrial DNA Part B 5: 271272.

Darriba, D., G. L. Taboada, R. Doallo and D. Posada. 2012. jModelTest 2: More models, new heuristics and parallel computing. Nature Methods 9: 772.

Greiner, S., P. Lehwark and R. Bock. 2019. OrganellarGenomeDRAW (OGDRAW) version 1.3.1: Expended toolkit for the graphical visualization of organellar genomes. Nucleic Acid Research 47: W59-W64.

Heo, K.-I., J. Park, H. Xi and J. Min. 2020. The complete chloroplast genome of Agrimonia pilosa Ledeb. isolated in Korea (Rosaceae): Investigation of intraspecific variations on its chloroplast genomes. Mitochondrial DNA Part B 5: 22642266.

Huang, P. and H. Werff. 2008. Neolitsea. In Flora of China. Vol. 7. Meinspermaceae through Capparaceae. Wu, Z. Y., P. H. Raven and D. Y. Hong (eds.), Science Press, Beijing and Missouri Botanical Garden, St. Louis, MO. Pp. 105-118.

Jo, H.-J., S.-K. Lee, H.-J. Lee, H.-Y. Kang, D.-H. Choi and I.-G. Choi. 2007. The dyeing properties of Hanji by Neolitsea sericea Koidz extracts. Journal of Korea TAPPI 39: 60-67. 
Katoh, K. and D. M. Standley. 2013. MAFFT multiple sequence alignment software version 7: Improvements in performance and usability. Molecular Biology and Evolution 30: 772-780.

Kim, T.-W. 2019. The Plants of Ulleungdo and Dokdo. Nature and Ecology, Seoul, 552 pp.

Kim, Y., K.-I. Heo and J. Park. 2019. The second complete chloroplast genome sequence of Pseudostellaria palibiniana (Takeda) Ohwi (Cryophyllaceae): Intraspecies variations based on geographical distribution. Mitochondrial DNA Part B 4: 1310-1311.

Koidzumi, G. 1926. Contributiones ad congnitionem Floræ Asiæ orientalis. Botanical Magazine (Tokyo) 40: 330-348.

Kwon, W., Y. Kim and J. Park. 2019. The complete chloroplast genome of Korean Marchantia polymorpha subsp. ruderalis Bischl. \& Boisselier: Low genetic diversity between Korea and Japan. Mitochondrial DNA Part B 4: 959-960.

Lee, J.-H. and B.-H. Choi. 2010. Distribution and northernmost limit on the Korean Peninsula of three evergreen trees. Korean Journal of Plant Taxonomy 40: 267-273.

Lee, J.-H., D.-H. Lee and B.-H. Choi. 2013. Phylogeography and genetic diversity of East Asian Neolitsea sericea (Lauraceae) based on variations in chloroplast DNA sequences. Journal of Plant Research 126: 193-202.

Lee, Y.-E., Y. Lee and S. Kim. 2021. A report of the second chloroplast genome sequence in Veronica nakaiana (Plantaginaceae), an endemic species in Korea. Korean Journal of Plant Taxonomy 51: 109-114.

Li, L., J. Li, J. G. Conran and X.-W. Li. 2007. Phylogeny of Neolitsea (Lauraceae) inferred from Bayesian analysis of nrDNA ITS and ETS sequences. Plant Systematics and Evolution 269: 203-221.

Ohashi, H., Y. Sasaki and K. Ohashi. 2006. The northern limit of distribution of Neolitsea sericea (Blume) Koidz. (Lauraceae) in the Pacific side of Japan. Journal of Japanese Botany 81: 248-249.
Park, J., Y. Bae, B.-Y. Kim, G.-H. Nam, J.-M. Park, B. Y. Lee, H.J. Suh and S.-H. Oh. 2021. The complete chloroplast genome of Campanula takesimana Nakai from Dokdo Island in Korea (Campanulaceae). Mitochondrial DNA Part B 6: 135-137.

Park, J., Y. Kim, H. Xi, Y. J. Oh, K. M. Hahm and J. Ko. 2019. The complete chloroplast genome of common camellia tree, Camellia japonica L. (Theaceae), adapted to cold environment in Korea. Mitochondrial DNA Part B 4: 1038-1040.

Qing, X., T. Zhao, R.-X. Guo, Y.-B. Wu, Q.-W. Shi, Y.-C. Gu and H. Kiyota. 2014. Chemical constituents of plants from the genus Neolitsea. Heterocyclic Communications 20: 61-75.

Song, Y., W.-B. Yu, Y. Tan, B. Liu, X. Yao, J. Jin, M. Padmanaba, J.-B. Yang and R. T. Corlett. 2017. Evolutionary comparisons of the chloroplast genome in Lauraceae and insights into loss events in the Magnoliids. Genome Biology and Evolution 9: 2354-2364.

Swofford, D. L. 2002. PAUP*: Phylogenetic analyses using parsimony (*and other methods), version 4.0b10. Sinauer, Sunderland, MA.

Yoo, S.-C., S.-H. Oh and J. Park. 2021. Phylogenetic position of Daphne genkwa (Thymelaceaceae) inferred from complete chloroplast data. Korean Journal of Plant Taxonomy 51: 171175.

Yoon, W.-J., J.-Y. Moon, J.-Y. Kang, G.-O. Kim, N. H. Lee and C.G. Hyun. 2010. Neolitsea sericea essential oil attenuates LPSinduced inflammation in RAW 264.7 macrophages by suppressing NF- $\kappa$ B and MAPK activation. Natural Product Communications 5: 1311-1316.

Yun, J.-H., K. Nakao, J.-H. Kim, S.-Y. Kim, C.-H. Park and B.-Y. Lee. 2014. Habitat prediction and impact assessment of Neolitsea sericea (Blume) Koidz. under climate change in Korea. Journal of Environmental Impact Assessment 23: 101-111. 